\title{
Design of Traveling Wave Location Method for Transmission Line Fault Based on EMD-TEO
}

\section{Juan Xia*}

Chengdu University of Technology, Chenghua District, Chengdu City 610059, Sichuan Province, China. E-mail: 1355871061@qq.com

Abstract: With the rapid development of the social economy and the continuous extension of Internet technology, China's power grid has entered the ranks of large-scale, high-voltage, and intelligent. The main purpose of the fault location of the transmission line is to eliminate hidden trouble and restore the fault line in time to ensure the safe and stable operation of the power system. With the advent of the smart grid, higher requirements are put forward for fault location accuracy, while the traditional wavelet transform and Hilbert-Huang transform have larger defects.

Therefore, this paper extensively analyses the generation and characteristics of fault traveling waves in transmission line fault, which proves that the traveling wave location method has higher location accuracy than the fault analysis method. Among them, the two-terminal traveling wave positioning method only uses the arrival time of the initial traveling wave, avoiding the principled defects and locating the dead zone of the single-terminal traveling wave positioning method, so the two-terminal traveling wave positioning method is generally used. The key of the two-terminal traveling wave location method is that it can accurately detect the arrival time of the initial traveling wave head. Although the Hilbert-Huang Transform (HHT) method can be used to detect the arrival time of the initial traveling wave head, the problem of inaccurate detection or failure of the wave head may arise when the instantaneous frequency of the IMF component decomposed by the Hilbert-Huang transform is used because of the mode aliasing in the empirical mode decomposition algorithm. Based on the above analysis, an empirical mode decomposition (EMD) combined with the Teager energy operator(TEO) is proposed for the traveling wave fault location of transmission lines. A large number of simulations prove that the EMD-TEO method in this paper can solve the problem of inaccuracy or failure of the HHT method using instantaneous frequency to detect the arrival time of wave head, and has higher fault location accuracy.

Keywords: Empirical Mode Decomposition; Teager Energy Operator; Fault Traveling Wave Location

\section{Introduction}

With the continuous extension of Internet technology, China's power grid has achieved large-scaled and intelligent development, and the requirements on the reliability, flexibility and availability of power supply of transmission system are also constantly improving. The purpose of transmission line fault-location is to find the fault point in time and remove it so as to restore the normal power supply of transmission line and ensure the reliable operation of the whole power system. The traditional solution to troubleshoot and restore power by means of manpower. However, with the introduction of smart power network, higher requirements are put forward for fault-location accuracy. Not only does manual

This is an open-access article distributed under the terms of the Creative Commons Attribution Non-Commercial License (http://creativecommons.org/licenses/by-nc/4.0/), which permits unrestricted non-commercial use, distribution, and reproduction in any medium, provided the original work is properly cited. 
labor consume time, but it also cannot meet the guarantee of reliability in relay protection. Nowadays, the highest priority is to study a new method of fault-location for transmission lines.

As is known to all, the fault transient traveling-wave is a kind of abrupt fault- signal which is not interfered by relay saturation and system oscillation like power frequency signal. Moreover, traveling-wave fault-location technique has some advantages that traditional location method cannot achieve, such as fast location speed and high location accuracy. In addition, it is not affected by factors like transition resistance, CT saturation and system oscillation. This has prompted the majority of researchers to constantly propose and improve new ideas for traveling-wave fault-location techniques ${ }^{[1-3]}$.

In recent years, with the continuous improvement of electronic technology, DSP technology, GPS satellite positioning system and so on have sprung up. On this basis, researchers have successively proposed various traveling-wave fault-location algorithms based on their own traveling-wave theory, and a large number of traveling-wave fault-location devices have also been put into use in the power system ${ }^{[4,5]}$. Based on the analysis of the single-ended unsynchronized traveling-wave fault-location algorithms, Hilbert-Huang transforms and Teager energy operator. This paper studies the traveling-wave fault-location techniques ${ }^{[6,7]}$. $\mathrm{In}^{[8]}$, the method of combining hardware detection and Hilbert-Huang transform software detection can achieve high accuracy detection of the arrival time of the faulty traveling wave head. On the basis of the original Hilbert-Huang trans- form method, the empirical mode decomposition method and Teager energy operator are combined to process the fault traveling-wave signal, so as to achieve precise location. Therefore, it is of positive significance to study traveling-wave fault-location techniques of transmission lines based on EMD-TEO method for the whole power system $^{[9,10]}$.

\section{Analysis of fault traveling wave in transmission line}

\subsection{Fault characteristics of fault traveling waves}

When the power system fails, the fault traveling wave will propagate in two opposite directions on the transmission line, and at the fault point and the system, because the wave impedance of some points is not continuous, the fault traveling wave will be at one of these points. It will reflect, refract, reflect, refract from time to time, until finally enter the steady-state process, which is called the wave process on the transmission line. When studying the wave process on the transmission line, the transmission line needs to be regarded as a distributed parameter loop. The transmission line can be regarded as the equivalent connection of many resistance, conductance, inductance, and capacitance elements. These four types of elements are called the distributed parameters of the line. The actual transmission line usually uses bipolar DC or three-phase AC transmission, so it is called a multi-conductor system, as shown in Figure 1.

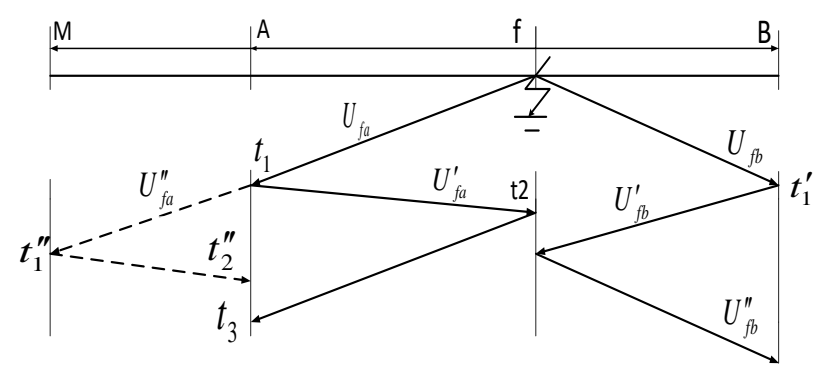

Figure 1. Transmission grid diagram of fault traveling wave of transmission line.

\subsection{Dispersion characteristics of fault trav- eling wave transmission}

In the power system, the fault traveling wave signal is a mixed-signal of some propagation modes. The traveling wave component in each propagation mode causes the difference in transmission characteristics due to the difference in frequency, which leads to the distortion of the fault traveling wave during the transmission process. This process is called the dispersion characteristic of the fault traveling wave. 
The traveling wave propagation coefficient is: $A(w)=e^{-\gamma l}=e^{-\alpha l} \cdot e^{-j \beta l}$

Where $\gamma=\alpha+j \beta$; $\alpha$ represents the attenuation constant; $\beta$ represents the phase constant, and both $\alpha$ and $\beta$ are frequency-dependent functions.

The phase velocity, which is the velocity of the in-phase point, is determined by the phase constant $\beta$, which is:

$V_{p}=\frac{w}{\beta}$

The values of $\alpha, \beta, \gamma$ and can be obtained from under each frequency component, so that the attenuation and phase shift process of each frequency component of the traveling wave during the propagation process can be clear, and then the dispersion of the traveling wave during propagation can be obtained.

\subsection{Phase-to-mode conversion of transmis- sion lines}

The essence of phase mode transformation is to use the eigenvector matrix of the coefficient matrix $\mathrm{ZY}$ or $\mathrm{YZ}$ as the mode transformation matrix, and at the same time diagonalize the matrices $\mathrm{ZY}, \mathrm{YZ}, \mathrm{Z}$, and $\mathrm{Y}$, to convert the coupled phase domain basic equation and wave equation into no coupling The basic equation of the model domain and the wave equation.

Because the Clark transformation matrix, symmetric component transformation matrix, and orthogonal transformation matrix all contain irrational numbers, and the computer itself is slow in processing irrational numbers, and it will produce errors, which will affect the processing results. Therefore, this paper selects the Karen Bell transformation matrix as the phase mode transformation matrix, the voltage traveling wave is selected as the phase-to-mode conversion object, so the following conversion equation is given:

$$
\left[\begin{array}{l}
\dot{U}_{0} \\
\dot{U}_{\alpha} \\
\dot{U}_{\beta}
\end{array}\right]=\frac{1}{3}\left[\begin{array}{ccc}
1 & 1 & 1 \\
1 & -1 & 0 \\
1 & 0 & -1
\end{array}\right]\left[\begin{array}{c}
\dot{U}_{a} \\
\dot{U}_{b} \\
\dot{U}_{C}
\end{array}\right]
$$

\section{The principle of fault location method based on EMD-TEO}

\subsection{Empirical mode decomposition (EMD)}

The signal $s(t)$ formed by the superposition of a tri- angle wave signal and a sine signal is decomposed by EMD to obtain two imf components and a residual component. The imf1 component corresponds to the sine signal, and the imf2 component corresponds to the triangle wave signal. The above decomposition process has extracted two mixed signals, so the decomposition result is ideal.

\subsection{Teager Energy Operator (TEO)}

TEO is a nonlinear operator that can effectively extract signal energy. Since TEO can reflect the changes in signal amplitude and frequency, it is also a feasible way to accurately locate the energy mutation point of the traveling wave signal. This is because when the fault traveling wave arrives, the frequency and amplitude of the signal will change exponentially, resulting in a sudden change point.

Suppose the discrete form of sine signal is:

$S_{n}=\operatorname{Acos}(\Omega n+\phi)$

Then its energy operator is expressed as:

$\psi\left(S_{n}\right)=S_{n}^{2}+S_{n+1} S_{n}=A^{2} \sin ^{2} \Omega$

Suppose a non-standard sinusoidal signal whose amplitude and frequency change with time is: $S_{n}=a_{n} \cos \varphi_{n}$

\subsection{Double-terminal traveling wave posi- tioning method based on EMD-TEO}

Since if only the Hilbert-Huang transform method is used to process the fault traveling wave signal, the positioning error will likely be too large or the positioning cannot be located. The new method first performs a phase-to-mode transformation on the voltage traveling wave component to obtain the decoupled line mode component; then use the EMD algorithm to process the decoupled line mode traveling wave component to obtain each eigenmode component; then use TEO to calculate the instantaneous energy value of the first eigenmode component; finally, according to the first, the energy mutation point determines the arrival time of the initial traveling wave head of the fault voltage, thereby calculating the location of the fault point on the transmission line.

\section{Research on traveling wave fault location algorithm of transmission line based on EMD-TEO}

\subsection{Simulation model building}


All simulation systems in this paper are a typical $110 \mathrm{kV}$ double-sided power line model with a total length of $250 \mathrm{~km}$. Points F1, F2, F3, F4, and F5 represent the distances from the M end 20 km, 90 km, 130 km, 180 $\mathrm{km}$, and $240 \mathrm{~km}$ respectively. Fault point in the area. Figure 2 is a simplified model. The line model in this paper uses a frequency correlation model, which can accurately represent the transient traveling wave process

The electromagnetic transient program ATP Draw is used to simulate the electromagnetic transient process fault of the line. The wire type selected in this paper is LGJ240/30, so its main parameters are shown in Table 1.

\begin{tabular}{cccc}
\hline & & \multicolumn{3}{c}{ phase } \\
\cline { 2 - 4 } Transmission line parameters & A & B & C \\
\hline Conductor inner diameter/cm & 0.8 & 0.8 & 0.8 \\
Conductor outer diameter/cm & 1.1 & 1.1 & 1.1 \\
DC resistance $/[1 /(\Omega \cdot \mathrm{km})]$ & 0.132 & 0.132 & 0.132 \\
The height of the conductor from the ground at the pole $/ \mathrm{m}$ & 28.3 & 28.3 & 28.3 \\
Split wire spacing/cm & 18.9 & 18.9 & 18.9 \\
Split wire separation angle $/{ }^{\circ}$ & 45 & 45 & 45 \\
Number of split wires & 180 & 180 & 180 \\
The distance between the tower and the center line $/ \mathrm{m}$ & 2 & 2 & 2 \\
\hline
\end{tabular}

Table 1. Transmission line parameters for simulation

Combining the above-mentioned understanding of ATP Draw and the known simplified model and line parameters of double-ended transmission lines, the follow- ing circuit simulation models can be built, as shown in Figure 2.

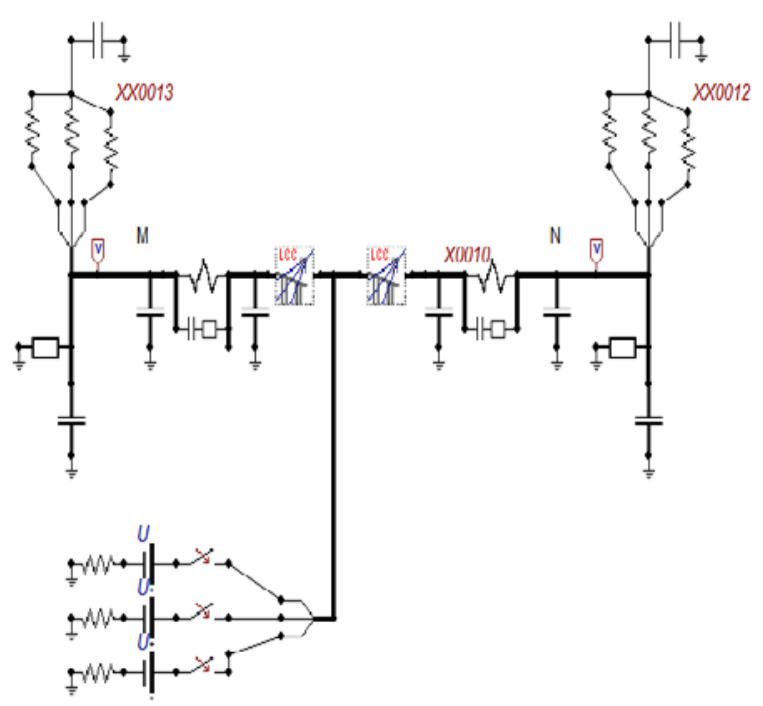

Figure 2. $110 \mathrm{kV}$ double-ended transmission line simulation model.

\subsection{Simulation analysis and algorithm veri- fication}

In the TEO energy curve graph, this article replaces the sampling time with the sampling point mode for a clear display. Then the time difference between two sampling points can be obtained by multiplying the dif- ference between the sampling points by the sampling period.

It can be concluded that when the $\mathrm{AB}$ two-phase grounding short circuit and the ABC three-phase short circuit occur at a distance of $180 \mathrm{~km}$ from the $\mathrm{M}$ terminal, the first mutation point in the TEO energy spectrum is located, and the sampling of the initial traveling wave 
head of the fault voltage reaching the $\mathrm{M}$ terminal and the $\mathrm{N}$ terminal can be obtained. The points are 802 and 435 respectively. Finally, according to the two-terminal traveling wave positioning formula mentioned above, the EMD-TEO method to locate the fault location is calculated as follows:

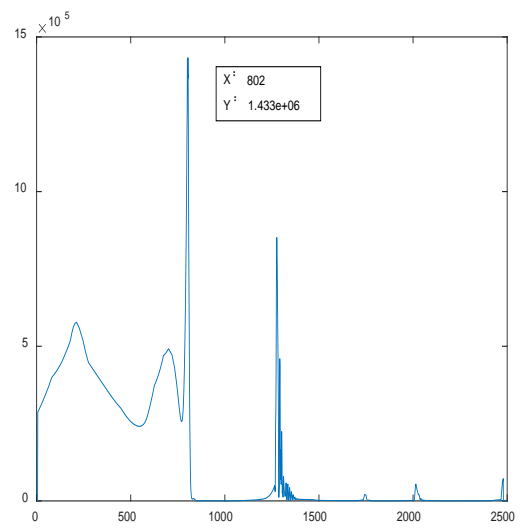

a. M end

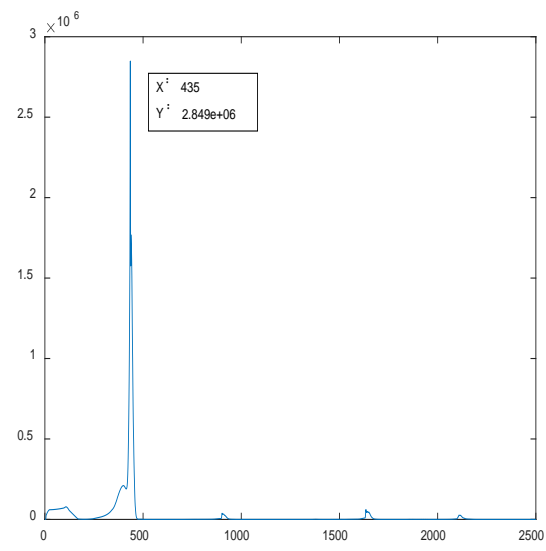

b. $\mathrm{N}$ end

Figure 3. Energy spectrum when phase A is grounded short circuit.

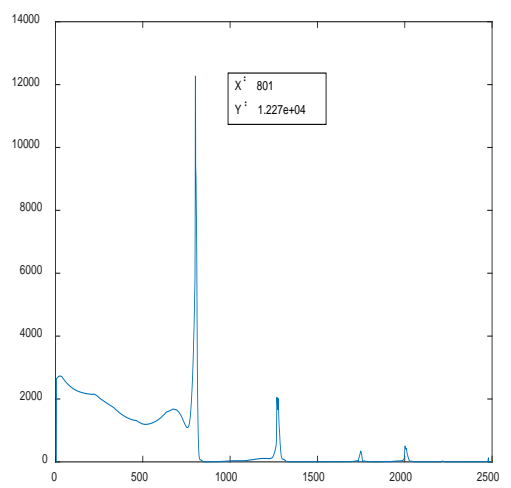

a. $\mathrm{M}$ end

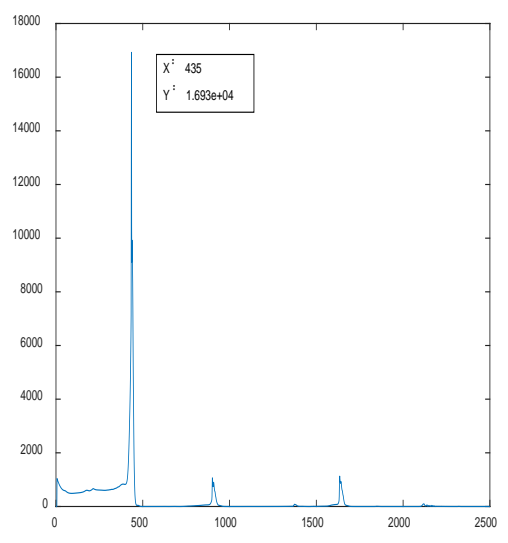

b. $\mathrm{N}$ end

Figure 4. Energy spectrum when three-phase short circuit.

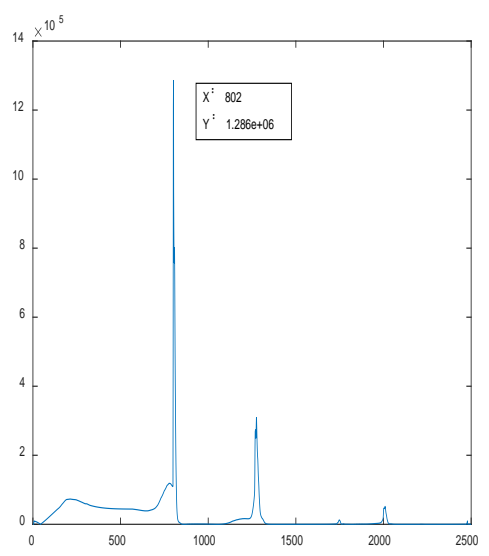

a. M end

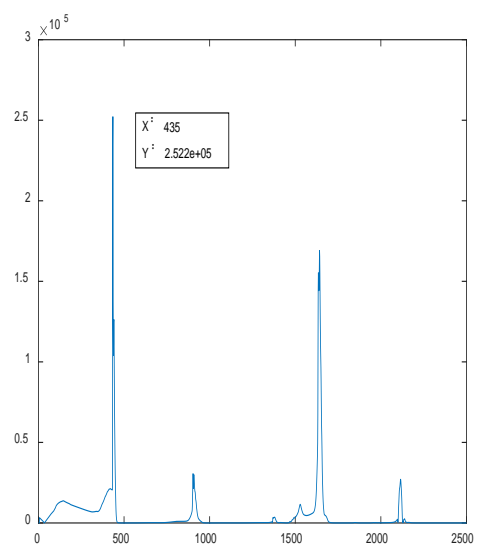

b. $\mathrm{N}$ end

Figure 5. Energy spectrum when $A B$ two phases are shorted to ground. 


\begin{tabular}{cccc}
\hline Fault type & $\begin{array}{c}\text { Traveling wave head } \\
\text { reaches the Sampling } \\
\text { point position } \mathbf{M} / \mathbf{N}\end{array}$ & $\begin{array}{c}\text { Distance } \\
/ \mathbf{k m}\end{array}$ & $\begin{array}{c}\text { Error } \\
/ \mathbf{k m}\end{array}$ \\
\hline $\mathrm{ABC}$ & $801 / 435$ & 180.085 & 0.085 \\
$\mathrm{ABg}$ & $801 / 435$ & 180.272 & 0.272 \\
$\mathrm{Ag}$ & $801 / 435$ & 180.085 & 0.085 \\
\hline
\end{tabular}

Table 2. Simulation results and errors

\subsection{Simulation result analysis}

The large number of simulation results presented above show that the new method proposed in this paper can not only solve the serious shortcomings of the Hilbert-Huang transform method in processing the eigenmode function but also accurate the arrival time of the faulty traveling wave head. The accuracy of fault location is greatly improved, and the error can be controlled at the level of 100 meters.

\section{Conclusion}

This article extensively analyzes the generation and characteristics of fault traveling waves when a transmission line fails. Since the positioning accuracy of the traveling wave positioning method is slightly better than that of the fault analysis method, the single-ended traveling wave positioning method or the double-ended traveling wave positioning method is generally selected. However, the double-ended traveling wave positioning method only uses the initial traveling wave head time, which avoids the problems of reflection wave attenuation and positioning dead zone. Therefore, the dual-terminal traveling wave positioning method is generally selected to improve the accuracy of positioning. Although the Hilbert-Huang transform method can be used to detect the arrival time of the initial traveling wave head of the fault, due to the existence of modal aliasing in the empirical mode decomposition algorithm, the Hilbert-Huang transform method is used to deal with the fault. Traveling wave signals are likely to cause excessive positioning errors.

To solve this problem, the EMD, TEO, and double-terminal traveling wave positioning methods to propose a method to determine the location of the fault point is combined in this paper. A large number of simulation results obtained in the previous chapters show that the new method proposed in this paper can not only solve the serious shortcomings of the Hilbert-Huang transform method in processing eigenmode functions, but also greatly improve the positioning accuracy, making the error can be controlled within a hundred meters.

\section{References}

1. Xi Y, Li Z, Zeng X, et al. Fault location based on travelling wave identification using an adaptive extended Kalman filter. IET Generation, Transmission \& Distribution 2018; 1314-1322.

2. Wu Z, Li Z, Qin X, et al. A novel double terminal traveling wave fault location method not influenced by wave speed. Transmission \& Distribution Conference \& Exposition: Asia and Pacific; 2009. p. $1-4$.

3. Deng F, Li X, Zeng X. Single-ended travelling wave protection algorithm based on full waveform in the time and frequency domains (in Chinese). IET Generation, Transmission \& Distribution 2018; 36803691.

4. Deng F, Li X, Zeng X, et al. Research on traveling wave fault location system based on high accuracy synchronous clock. 2015 5th International Conference on Electric Utility Deregulation and Restructuring and Power Technologies (DRPT); 2015. p. 1147-1151.

5. Magnago FH, Abur A. Fault location using wavelet. IEEE Trans on Power Delivery 1998; 13(4): 1475-1480.

6. Huang NE, Shen Z, Long SR, et al. The empirical mode decomposition and the Hilbert spectrum for nonlinear and non-stationary time series analysis. Proc. R Soc Lond A 1998; 454(21): 903-995.

7. Huang NE, Shen Z, Long SR. A new view of nonlinear waves: the Hilbert spectrum. Annual Review of Fluid Mechanics 1999; 31(1): 417-457.

8. Liang J, Zhou J. Application of multi-waves theory for the fault location in transmission line. Power System Protection and Control 2008; 36(13): 26-32.

9. Zhao Z, Ma C. An intelligent system for noninvasive diagnosis of coronary artery disease with EMD-TEO and BP neural network. IEEE; 2009.

10. Wang N, Liu D, Liao Q, et al. Identification of the dominant inertial mode based on EMD-TEO and signal energy method. Transactions of China Electrotechnical Society 2012; 27(6): 198-204. 